\title{
Sperm telomere length: Diagnostic and prognostic biomarker in male infertility (Review)
}

\author{
SAIRA AMIR ${ }^{1 *}$, ELENA VAKONAKI $^{2 *}$, KONSTANTINA TSIMINIKAKI $^{2}$, \\ MANOLIS N. TZATZARAKIS ${ }^{2}$, VASILIKI MICHOPOULOU ${ }^{2}$, MATTHAIOS FLAMOURAKIS ${ }^{3}$, \\ KATERINA KALLIANTASI ${ }^{2}$, VASILIKI KARZI ${ }^{2}$, PERSEFONI FRAGKIADAKI ${ }^{2}$, ELISAVET A. RENIERI ${ }^{2}$, \\ DIMITRIS TSOUKALAS ${ }^{4}$, MARIA THANASOULA ${ }^{3}$, EVANGELIA SARANDI $^{3}$, \\ GEORGE SAKELLARIS $^{5}$, ANTONIOS MAKRIGIANNAKIS ${ }^{6}$, CHARITINI NEPKA $^{7}$, \\ DEMETRIOS A. SPANDIDOS ${ }^{8}$ and ARISTIDIS TSATSAKIS ${ }^{2}$
}

\author{
${ }^{1}$ Department of Biosciences, COMSATS Institute of Information Technology Islamabad, Islamabad, \\ Islamabad Capital Territory 45550, Pakistan; ${ }^{2}$ Laboratory of Toxicology, Medical School, University of Crete, \\ 71003 Heraklion; ${ }^{3}$ Venizeleio General Hospital, Department of Surgery, 71409 Heraklion; ${ }^{4}$ Metabolomic Medicine Clinic, \\ 10674 Athens; ${ }^{5}$ Department of Pediatric Surgery, University Hospital of Heraklion; ${ }^{6}$ Department of Obstetrics and \\ Gynecology, School of Medicine, University Hospital of Heraklion, University of Crete, 71003 Heraklion; \\ ${ }^{7}$ Department of Cytopathology, University Hospital of Larissa, 41110 Larissa; ${ }^{8}$ Laboratory of \\ Clinical Virology, School of Medicine, University of Crete, 71003 Heraklion, Greece
}

Received November 22, 2019; Accepted December 31, 2019

DOI: $10.3892 /$ wasj.2020.31

\begin{abstract}
Telomere length is an important biomarker in a number of diseases, including male infertility. A decreased telomere length has been reported in several diseases and telomere shortening may occur due to aging, decreased telomerase activity, oxidative stress or cell division. In recent years, several studies have indicated that males with infertility have a shorter sperm telomere length than fertile males. Sperm telomere shortening is associated with male infertility through several mechanisms, including the apoptosis of spermatozoa, decreased motility, low sperm count, incorrect chromosomal pairing and movement during meiosis, and failed fertilization. The aim of this review was to compile current findings on sperm telomere length and discuss findings to compare sperm from infertile males with that of fertile males. Several studies reported shortening of sperm telomeres associated with infertility. Thus, sperm telomere length can be used as biomarker for the diagnosis and prognosis of male infertility, since fertile males have longer telomeres and the length decreases with age. However, there is no specific telomere length that is set as standard/recommended length.
\end{abstract}

Correspondence to: Professor Aristidis Tsatsakis, Laboratory of Toxicology, Medical School, University of Crete, Voutes, 71003 Heraklion, Greece

E-mail: tsatsaka@uoc.gr

*Contributed equally

Key words: male infertility, telomere length, biomarker, sperm, diagnosis, prognosis

\section{Contents}

1. Introduction

2. Telomere length in sperm cells

3. Telomere length and male infertility

4. Role of sperm telomere in diagnosis and prognosis of male infertility

5. Conclusions

\section{Introduction}

Telomeres are highly conserved non-coding tandem repeats (5'-TTAGGG-3') located at the extreme end of chromosomes, coated by a complex of 6 proteins, termed the 'shelterin complex' and they are essential for genomic stability and integrity. The telomeric length of human germ cells $(10-20 \mathrm{~kb})$ is longer compared to that of human somatic cells $(5-10 \mathrm{~kb})(1)$. Telomeric length is heritable and varies with sex, as females have longer telomeres due to the presence of estrogen (2). However, the difference is insignificant between males and females at birth (3). In the majority of cases, the telomeric length of somatic cells reduces with age, as well as during each cell division (4). A number of factors, such as the environment, genetics, infection, lifestyle, oxidative stress, telomere uncapping and psychological stress are associated with telomere shortening (5). A marked decrease in telomeric length leads to chromosomal instability, resulting in apoptosis, senescence or tumorigenesis.

Telomerase, the enzyme responsible for telomere replication, plays a crucial role on telomeric length. It is an RNA-dependent DNA polymerase with a functional RNA component, hTERC, the template for telomere synthesis and a catalytic subunit hTERT, 
that is responsible for telomerase activity (6). Importantly, active telomerase compensates for telomere length shortening that is present in various age-related and chronic diseases, while it also plays a critical role in maintaining spermatogenesis in germ line cells, an important factor for male fertility (7).

As regards spermatogenesis, it is a procedure that occurs inside the seminiferous tubules of the testes, depending on human spermatogonial stem cells, and it is the result of a mitotic and two meiotic divisions. Inside the seminiferous tubule epithelium there is a basement membrane consisting of undifferentiated spermatogonia and differentiating type $B$ spermatogonia. The latter produce primary spermatocytes which follow meiotic division and migrate from the membrane. In addition, the following meiotic divisions and spermiogenesis result in the production of spermatids, the secondary spermatocytes and differentiated spermatozoa, which are delivered into the lumen of the seminiferous tubules. Spermatogenesis is completed in approximately 74-120 days, whereas millions of spermatozoa are released by the male testes daily.

As regards infertility, this has become a serious public health concern affecting millions of couples globally (8). Male infertility is the inability of a male to cause pregnancy in a fertile female after 12 months of regular unprotected intercourse (9). Approximately $50 \%$ of male infertility cases are due to environmental, behavioral and nutritional factors (10), spermiogenic defects (11), hormonal deregulation (12), sexual disorders and reproductive tract obstruction (13). However, the a etiology of approximately half of the cases of male infertility remains unexplained (idiopathic) (14), even though recent studies have reported that oxidative stress, DNA damage (15) and telomere shortening are attributed to idiopathic infertility (16).

The diagnosis of male infertility is generally based on standard semen analysis (17), a physical examination, personal and family history, ultrasound analysis and hormonal evaluation $(18,19)$. However, these methods are not sufficient for diagnosis, particularly in infertile males with normal semen parameters (idiopathic infertility) (20). Therefore, there is an increasing need for further diagnostic techniques that can also cover the a etiology and pathogenic profile of idiopathic infertile males. This fact combined with the results of recent studies that have reported shortening of sperm telomere length in male infertility, led us to compose this review in order to highlight the significance of sperm telomere length as a prognostic and diagnostic biomarker in male infertility (21). As illustrated in Table I, in the majority of studies, for the determination of telomeres, qPCR and Q-FISH have been used. In addition, Fig. 1 illustrates he advantages and disadvantages of both methods.

\section{Telomere length in sperm cells}

While women are born with a determined number of oocytes for the rest of their lives, in males, spermatogenesis is a dynamic and ongoing progress from puberty until death. Sperm is developed from spermatogonial stem cells in the seminiferous tubules of testis following a mitotic and two meiotic divisions. Millions of spermatozoa are produced by the male testes daily and spermatogenesis needs about 74-120 days to be completed (22).

Despite the fact that telomere length varies among different cells and organs (23), it is well known that human germ cells have longer telomeres than human somatic cells (24). It is a paradox that sperm telomeres, despite being longer, seem to elongate with age compared with somatic cells and the exact mechanisms of this process remain unclear $(25,26)$. One explanation is that molecular resistance against the aging process may be genetically programmed (26) and since an estimated 100 million sperm cells are produced daily, special telomere maintenance mechanisms are required to avoid rapid telomere shortening (27). Along these lines, it has been reported that there is high telomerase activity in the testes, leading to the gradual and progressive lengthening of sperm telomeres with age, rather than simply maintaining a stable length. This could mean that telomerase expression favors the long telomeres against the shorter which is not true, according to studies reporting that in general, telomerase lengthens preferentially shorter telomeres. Therefore, a plausible explanation is that sperm stem cells with shorter telomere length are disproportionately led to death with age, as a selective cellular process for telomere length maintenance $(25,27)$. This is supported by the fact that, according to a series of studies, for each additional year of paternal age at the time of birth, an increase of $17.7 \mathrm{bp}$ is observed in telomere length of the offspring's leucocytes (28) and sperm cells (27). However, despite this fact, the offspring of older fathers inherit longer telomeres; the greater the age of the father, the greater the danger of spontaneous germ cell mutations and as a consequence, of rare diseases in the offspring, such as achondroplasia and craniosynostosis. This is due to the higher number of replication cycles in terms of spermatogenesis, that corresponds to a higher error possibility during that process (29).

\section{Telomere length and male infertility}

In contrast to the above, even if sperm telomere length increases with age, researchers have suggested that male fertility in the late thirties and particularly after forties is reduced, significantly increasing the possibility of infertility if the woman is also of advanced age (30). It is important to mention though, that sperm telomere length shortening is not only derived from aging itself, but may also be a consequence of age-related diseases or oxidative stress that causes dysfunctions, which are a clear indication of infertility, but cannot be diagnosed by existing diagnostic procedures.

Importantly, human telomeric length of both somatic and germ cells plays a crucial role in human development and reproduction, as a shorter telomere length has been associated with unexplained frequent (31) mortality and reproductive aging (32). More specifically, a shorter telomere length in germ cells is associated with a number of reproductive complications, including infertility (33), failed fertilization, embryonic fatality, reduced lifespan and viability (34), cell cycle arrest, genomic instability, gamete apoptosis and frequent miscarriage (35-38). In agreement with this, it has been shown that infertile males have a shorter sperm telomere length compared to fertile males $(15,39)$. This could explain the fact that sperm with a shorter telomere length cannot fertilize an egg (form a zygote) due to the critical role of the sperm telomeric site in pronucleus formation and meiosis. Several studies have reported a strong association between sperm telomere length and sperm count $(5,17,21,40)$, as well as, the age of the parents at conception $(26,41)$. In addition, freshly ejaculated sperm must acquire certain characteristics, 
Table I. Studies associating telomere length with infertility.

Method used for the measurement

Authors/(Refs.), year

Kimura et al (25), 2008

Eisenberg et al (27), 2012

Thilagavathi et al (31), 2013

Mishra et al (33), 2016

Herrera et al (34),1999

Baird et al (36), 2006

Cariati et al (37), 2016

Torra-Massana et al (38), 2018

Yang et al (40), 2015

qPCR

- Southern blot analysis

- STELA PCR

qPCR

qPCR

of telomeric length

- Southern blot analysis

- Q-FISH

- flow-FISH

Monochrome multiplex quantitative PCR assay

qPCR method (correlation with DFI and ROS levels)

qPCR

qFISH

qPCR
Results

Offspring telomere length depends on paternal age

Longer sperm telomere length in older men indicates that the capability of reproduction could be extended STL is associated with infertility, although there was no association found between TL and ROS levels or sperm DNA damage

Shorter TL is connected to infertility. In addition, seminal reactive oxygen species (ROS) as well as 8-Isoprostane levels were higher in infertile men Failed fertilization, embryonic fatality, reduced lifespan and viability are related to short STL

Short STL may cause aberrant meiosis which leads to the production of aneuploid sperm

Shorter STL is associated with infertility, oligospermia and chromosomal abnormality In contrast with the literature, the multilevel biochemical, clinical analysis confirmed that the effect of STL on fertilization was not significant $(\mathrm{P}>0.05)$

No significant positive association between paternal age and STL at the time of conception sperm with longer TL could be obtained following density gradient centrifugation, in order to ameliorate the efficacy in assisted reproduction techniques STL is longer than leukocyte telomere length. STL in oligozoospermic males is significantly shorter than STL in normozoospermic males; a significant positive association between maternal age and both leukocyte and sperm telomere length and a significant positive association was found between paternal age and STL in the offspring

STL, sperm telomere length.

which make it competent to fertilize an egg (42). The quality of sperm depends on several parameters, such as sperm count, motility, vitality, reactive oxygen species (ROS) levels, DNA fragmentation index (DFI) and sperm telomere length. Sperm telomere length is essential during spermatogenesis, fertilization, pronucleus formation and meiosis, although the exact mechanisms of sperm telomere length regulation in male infertility are not yet fully understood. Sperm telomere length is directly associated with vitality, protamination and progressive motility, and is negatively associated with DNA fragmentation.

The link between sperm telomere length and its consequences in male fertility is possibly the increased oxidative stress. Oxidative stress is extremely damaging to hematopoietic stem cells and has been shown to be responsible for the dysfunction and aging of both somatic and germ cells (43). Severe oxidative stress is one of the major factors responsible for male infertility (44) and telomere shortening $(45,32)$. Telomeres are rich in residues (guanines) that are susceptible to oxidative stress leading to increased sperm DNA damage, which consequently reduces the quality of the sperm, resulting in infertility. However, Thilagavathi et al found no correlation between the levels of ROS, sperm DNA damage and sperm telomere length (31), while another study reported that mild oxidative stress played a role in sperm telomere lengthening (32), suggesting that mild oxidative stress may play a role in maintaining the genomic stability of the gamete.

\section{Role of sperm telomere in diagnosis and prognosis of male infertility}

Despite the variation of sperm telomere length between individuals, it can provide information on male infertility. As 

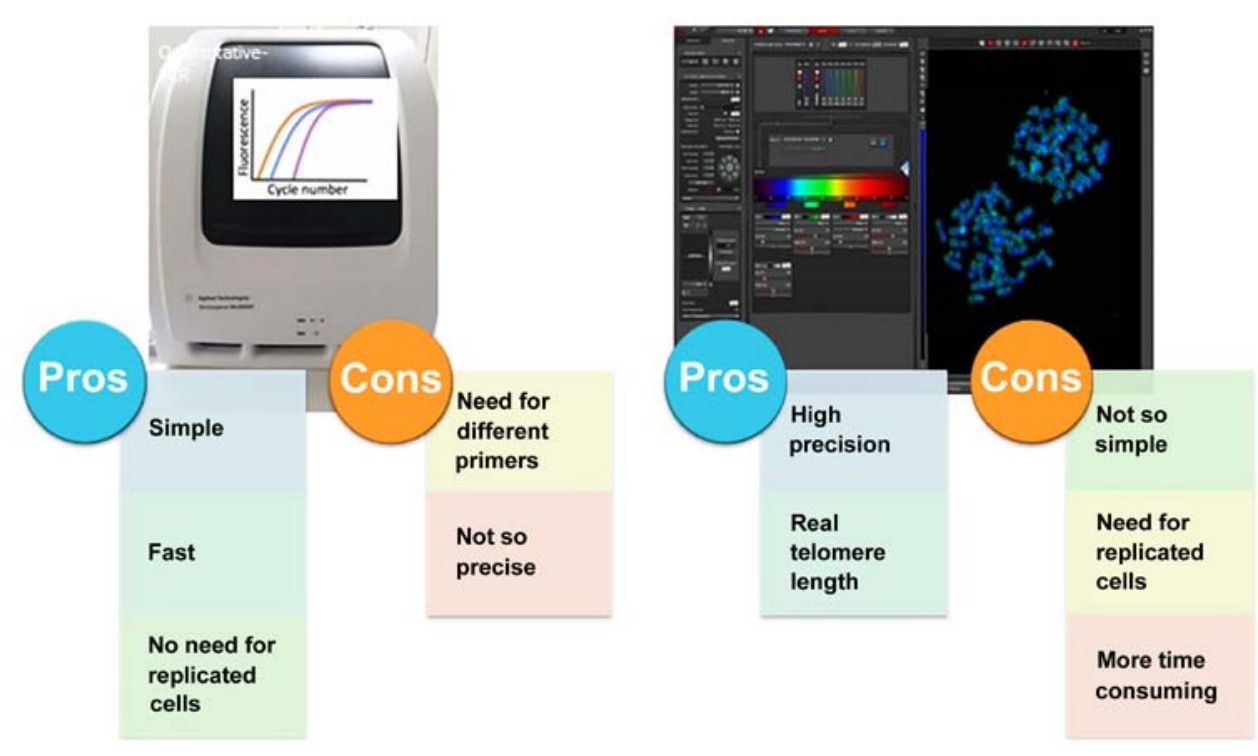

Figure 1. Advantages and disadvantages of qPCR and Q-FISH.

already mentioned, previous studies have indicated that the sperm telomere length of fertile males is significantly higher compared to that of infertile males $(5,31,33-35,39)$. Thus, sperm telomere length can provide insight (information) on male fertility, since a shortened telomere may be an indication of impaired spermatogenesis, which can lead to a low sperm count, error(s) in chromosomes segregation and imbalanced gametes. Moreover, another study reported a strong association between sperm telomere length and sperm count; i.e., males with longer sperm telomeres tend to have a good sperm count than those with shorter sperm telomeres (38). However, the role of a short telomere length of sperm cells in infertility remains under investigation. If the age factor and other systemic pathologies affecting telomere length are obliterated from the parameters of a study, it seems that men with oligozoospermia in contrary with those that are normozoospermic, have a shorter telomere sperm length (38). Moreover, males with idiopathic infertility have a shorter sperm telomere length, even though they are normozoospermic, compared to fertile males (30).

Since sperm telomere length is strongly associated with sperm count, motility and decreased DNA fragmentation, sperm telomeres can be used as a biomarker for the diagnosis and prognosis of male infertility. Importantly, sperm telomere length can be also used to assess sperm quality during assisted reproductive techniques (ART), suggesting that it may be used as one of the criteria for sperm selection during ART. For example, according to previous research, oligozoospermic males with a shorter sperm telomere length pass on a shorter telomere length to their offspring (30). Therefore, even though infertility treatment outcome determination is generally based on the ability to conceive or bear a child, semen quality, also based on sperm telomere length, can be used as a parameter to estimate the effectiveness of a treatment.

\section{Conclusions}

Male infertility accounts for approximately half of the total number of infertility cases. Thus far, semen parameter analysis is the most commonly used method for the diagnosis of male infertility, as well as for the determination of the medication outcome (i.e., the success of treatment). However, this method cannot be used for infertile males with normal semen parameters. Therefore, the use of sperm telomere length may be relevant to both the diagnosis and prognosis of idiopathic infertility.

\section{Acknowledgements}

Not applicable.

\section{Funding}

This work was supported by the Toxplus S.A. and the special account for research (ELKE) of the University of Crete (KA 3464, 3963, 3962).

\section{Availability of data and materials}

Not applicable.

\section{Authors' contributions}

SA, EV, KT, MNT, VM, MF, KK, VK, PF, EAR, DT, MT, $\mathrm{ES}, \mathrm{GS}, \mathrm{AM}$ and $\mathrm{CN}$ contributed to the writing of the manuscript and assisted with the literature search for this review article. EV, MNT DAS and AT contributed to the conception and design of the study and to the proofreading and editing of the manuscript. All authors have taken the responsibility for publishing this review article and all authors have read and approved the final manuscript.

\section{Ethics approval and consent to participate}

Not applicable.

\section{Patient consent for publication}

Not applicable. 


\section{Competing interests}

DAS is the Managing Editor of the journal, but had no personal involvement in the reviewing process, or any influence in terms of adjudicating on the final decision, for this article. The other authors declare that they have no competing interests.

\section{References}

1. Vakonaki E, Tsiminikaki K,Plaitis S, Fragkiadaki P, Tsoukalas D, Katsikantami I, Vaki G, Tzatzarakis MN, Spandidos DA and Tsatsakis AM: Common mental disorders and association with telomere length. Biomed Rep 8: 111-116, 2018.

2. Ruiz-Larrea MB, Leal AM, Martín C, Martínez R and Lacort M: Antioxidant action of estrogens in rat hepatocytes. Rev Esp Fisiol 53: 225-229, 1997.

3. Okuda K, Bardeguez A, Gardner JP, Rodriguez P, Ganesh V, Kimura M, Skurnick J, Awad G and Aviv A: Telomere length in the newborn. Pediatr Res 52: 377-381, 2002.

4. Blackburn EH: Structure and function of telomeres. Nature 350: 569-573, 1991.

5. Thilagavathi J, Venkatesh S and Dada R: Telomere length in reproduction. Andrologia 45: 289-304, 2013.

6. Biron-Shental T, Wiser A, Hershko-Klement A, Markovitch O, Amiel A and Berkovitch A: Sub-fertile sperm cells exemplify telomere dysfunction. J Assist Reprod Genet 35: 143-148, 2018.

7. Oshimura M and Barrett JC: Multiple pathways to cellular senescence: Role of telomerase repressors. Eur J Cancer 33: 710-715, 1997.

8. Sharpe RM: Sperm counts and fertility in men: A rocky road ahead. Science \& Society Series on Sex and Science. EMBO Rep 13: 398-403, 2012.

9. Gnoth C, Godehardt E, Frank-Herrmann P, Friol K, Tigges J and Freundl G: Definition and prevalence of subfertility and infertility. Hum Reprod 20: 1144-1147, 2005.

10. Singh K and Jaiswal D: Human male infertility: A complex multifactorial phenotype. Reprod Sci 18: 418-425, 2011

11. Yan W: Male infertility caused by spermiogenic defects: Lessons from gene knockouts. Mol Cell Endocrinol 306: 24-32, 2009.

12. McLachlan RI, Wreford NG, O'Donnell L, de Kretser DM and Robertson DM: The endocrine regulation of spermatogenesis: Independent roles for testosterone and FSH. J Endocrinol 148 : 1-9, 1996.

13. De Kretser DM and Baker HW: Infertility in men: Recent advances and continuing controversies. J Clin Endocrinol Metab 84: 3443-3450, 1999.

14. Esteves SC: A clinical appraisal of the genetic basis in unexplained male infertility. J Hum Reprod Sci 6: 176-182, 2013.

15. Shamsi MB, Venkatesh S, Pathak D, Deka D and Dada R Sperm DNA damage \& oxidative stress in recurrent spontaneous abortion (RSA). Indian J Med Res 133: 550-551, 2011.

16. Rocca MS, Speltra E, Menegazzo M, Garolla A, Foresta C and Ferlin A: Sperm telomere length as a parameter of sperm quality in normozoospermic men. Hum Reprod 31: 1158-1163, 2016.

17. Franken DR and Oehninger S: Semen analysis and sperm function testing. Asian J Androl 14: 6-13, 2012

18. Jungwirth A, Giwercman A, Tournaye H, Diemer T, Kopa Z, Dohle G and Krausz C; European Association of Urology Working Group on Male Infertility: European Association of Urology guidelines on Male Infertility: The 2012 update. Eur Urol 62: 324-332, 2012.

19. Foresta C, Ferlin A, Gianaroli L and Dallapiccola B: Guidelines for the appropriate use of genetic tests in infertile couples. Eur J Hum Genet 10: 303-312, 2002.

20. Quaas A and Dokras A: Diagnosis and treatment of unexplained infertility. Rev Obstet Gynecol 1: 69-76, 2008.

21. Vasilopoulos E, Fragkiadaki P, Kalliora C, Fragou D, Docea AO, Vakonaki E, Tsoukalas D, Calina D, Buga AM, Georgiadis G, et al: The association of female and male infertility with telomere length (Review). Int J Mol Med 44: 375-389, 2019.

22. Amann RP: The cycle of the seminiferous epithelium in humans: A need to revisit? J Androl 29: 469-487, 2008.

23. Bekaert S, Derradji $\mathrm{H}$ and Baatout S: Telomere biology in mammalian germ cells and during development. Dev Biol 274 15-30, 2004.
24. Aston KI, Hunt SC, Susser E, Kimura M, Factor-Litvak P, Carrell D and Aviv A: Divergence of sperm and leukocyte age-dependent telomere dynamics: Implications for male-driven evolution of telomere length in humans. Mol Hum Reprod 18: 517-522, 2012.

25. Kimura M, Cherkas LF, Kato BS, Demissie S, Hjelmborg JB, Brimacombe M, Cupples A, Hunkin JL, Gardner JP, Lu X, et al: Offspring's leukocyte telomere length, paternal age, and telomere elongation in sperm. PLoS Genet 4: e37, 2008.

26. Sharma R, Agarwal A, Rohra VK, Assidi M, Abu-Elmagd M and Turki RF: Effects of increased paternal age on sperm quality, reproductive outcome and associated epigenetic risks to offspring. Reprod Biol Endocrinol 13: 35, 2015.

27. Eisenberg DT, Hayes MG and Kuzawa CW: Delayed paternal age of reproduction in humans is associated with longer telomeres across two generations of descendants. Proc Natl Acad Sci USA 109: 10251-10256, 2012.

28. Prescott J, Du M, Wong JY, Han J and De Vivo I: Paternal age at birth is associated with offspring leukocyte telomere length in the nurses' health study. Hum Reprod 27: 3622-3631, 2012.

29. Crow JF: The origins, patterns and implications of human spontaneous mutation. Nat Rev Genet 1: 40-47, 2000.

30. Kühnert B and Nieschlag E: Reproductive functions of the ageing male. Hum Reprod Update 10: 327-339, 2004.

31. Thilagavathi J, Kumar M, Mishra SS, Venkatesh S, Kumar R and Dada R: Analysis of sperm telomere length in men with idiopathic infertility. Arch Gynecol Obstet 287: 803-807, 2013.

32. Shay JW: Role of Telomeres and Telomerase in Aging and Cancer. Cancer Discov 6: 584-593, 2016.

33. Mishra S, Kumar R, Malhotra N, Singh N and Dada R: Mild oxidative stress is beneficial for sperm telomere length maintenance. World J Methodol 6: 163-170, 2016.

34. Herrera E, Samper E, Martín-Caballero J, Flores JM, Lee HW and Blasco MA: Disease states associated with telomerase deficiency appear earlier in mice with short telomeres. EMBO J 18: 2950-2960, 1999.

35. Kalmbach KH, Fontes Antunes DM, Dracxler RC, Knier TW, Seth-Smith ML, Wang F, Liu L and Keefe DL: Telomeres and human reproduction. Fertil Steril 99: 23-29, 2013.

36. Baird DM, Britt-Compton B, Rowson J, Amso NN, Gregory L and Kipling D: Telomere instability in the male germline. Hum Mol Genet 15: 45-51, 2006.

37. Cariati F, Jaroudi S, Alfarawati S, Raberi A, Alviggi C, Pivonello R and Wells D: Investigation of sperm telomere length as a potential marker of paternal genome integrity and semen quality. Reprod Biomed Online 33: 404-411, 2016.

38. Torra-Massana M, Barragán M, Bellu E, Oliva R, Rodríguez A and Vassena R: Sperm telomere length in donor samples is not related to ICSI outcome. J Assist Reprod Genet 35: 649-657, 2018.

39. Ferlin A, Rampazzo E, Rocca MS, Keppel S, Frigo AC, De Rossi A and Foresta C: In young men sperm telomere length is related to sperm number and parental age. Hum Reprod 28: 3370-3376, 2013

40. Yang Q, Zhang N, Zhao F, Zhao W, Dai S, Liu J, Bukhari I, Xin H, Niu W and Sun Y: Processing of semen by density gradient centrifugation selects spermatozoa with longer telomeres for assisted reproduction techniques. Reprod Biomed Online 31: 44-50, 2015.

41. Sartorius GA and Nieschlag E: Paternal age and reproduction. Hum Reprod Update 16: 65-79, 2010.

42. Jin SK and Yang WX: Factors and pathways involved in capacitation: How are they regulated? Oncotarget 8: 3600-3627, 2017.

43. Ito K, Hirao A, Arai F, Takubo K, Matsuoka S, Miyamoto K, Ohmura M, Naka K, Hosokawa K, Ikeda Y, et al: Reactive oxygen species act through p38 MAPK to limit the lifespan of hematopoietic stem cells. Nat Med 12: 446-451, 2006.

44. Bisht S, Faiq M, Tolahunase M and Dada R: Oxidative stress and male infertility. Nat Rev Urol 14: 470-485, 2017.

45. Oikawa S and Kawanishi S: Site-specific DNA damage at GGG sequence by oxidative stress may accelerate telomere shortening. FEBS Lett 453: 365-368, 1999.

This work is licensed under a Creative Commons Attribution-NonCommercial-NoDerivatives 4.0 International (CC BY-NC-ND 4.0) License. 